\title{
E-commerce System of Community based on J2EE
}

\author{
Hongqing Liu \\ Hunan vocational college of modern logistics,Changsha, Hunan,410131
}

Keywords: e-government, J2EE, System design, Enterprise informatization, Examples.

\begin{abstract}
China and the whole world, e-commerce has had a tremendous impact on social economy and people's lives. The traditional Chinese government and enterprises, which are relatively backward in informatization, are facing new challenges and opportunities for the development of communities and other communities in china. Firstly, the characteristics and development trend of electronic commerce concept, with the combination of community and the current form of domestic post enterprises, objective analysis of the community in the field of electronic commerce's advantages and disadvantages, and points out the necessity of community development of e-commerce, the use of J2EE technology to support the construction of the community e-commerce system based on J2EE". The system takes the community as the object, and gives a complete set of community e-commerce solution. This paper gives a detailed analysis, design and implementation of the "one card subsystem" in the system, and illustrates the application of e-commerce in community business through a specific example.
\end{abstract}

\section{Introduction}

Electronic Commerce (Electronic Commerce EC) is based on the Internet of the new business model, breakthrough time and space limitation, completely changed the traditional commercial mode, to create new business opportunities. According to the definition of the World Trade Organization electronic commerce special report: electronic commerce is the production, management, sales and distribution activities through the computer network, it not only refers to transactions based on the activities of Internet (the concept of electronic business system), also refers to all the use of electronic information technology to solve problems quickly, reduce costs, and expand publicity add value and create business opportunities for business activities, including through the network from raw materials procurement to query, production, storage and electronic payment, customer service and a series of business activities.

The use of e-commerce enterprises can not only through direct contact with tens of thousands of new users of the network, and to deal with them, radically streamlining the commercial links, reduce operating costs, improve operational efficiency, increase enterprise profit. It also can communicate with other trade partners all over the world at any time, enhance the cooperation among enterprises, and improve the competitiveness of products. Compared with the traditional business mode, e-commerce has the following characteristics.

(1) reduced circulation: electronic commerce does not require wholesalers, stores and shopping malls, customers through the network directly from the merchants to order products.

(2) save shopping time: customers can purchase satisfied goods through the network without leaving the house.

(3) to accelerate the circulation of funds: e-commerce turnover without between outside of the bank customers, wholesalers, shopping malls, and through the network within the bank account directly, greatly accelerate the capital turnover rate, but also reduce the commercial disputes.

(4) enhance the communication between customers and manufacturers: customers can express their own requirements through the network, order products, manufacturers can quickly understand the user needs, avoid waste of product production.

(5) stimulate the cooperation and competition among enterprises: enterprises can understand the competitors' product performance and price, sales volume and other information through the 
network, so as to promote the enterprise transformation technology, improve product competitiveness.

\section{2 . The development trend of e-commerce}

With the rapid development of computer communication technology, modern transportation network and the popularity of e-commerce, especially after China's accession to the WTO, the traditional Chinese community business has been greatly challenged. In the logistics business, the major foreign companies (such as TNT, FedEx, UPS) have been added China logistics market competition, competition for market share; in the information business, community is facing many Internet companies in the competition; financial business is facing the competition of commercial banks and so on. The main business community had the advantages of a traditional are facing severe challenges to the sustainable development of the business community, and the community industry are in urgent need to find a new economic growth point and new management and service mode, develop a broader space for development, this is the electronic commerce [4].

Postal enterprises veteran Ma Fengjiong through the analysis of the community is the external environment and internal conditions and other factors in the community "business strategy" in one book, the community's mission and business areas, business ideas and strategic objectives. He believes that in today's information age, the community needs to be based on integrated computer networks, community physical delivery network and community financial network as the foundation of e-commerce business. E-commerce is the main means for the community to further provide services for the community. The promotion of e-commerce in community enterprises will greatly promote the pace of community development. Deputy director of the State Council on the "male warrior community development" from the practice of electronic commerce Chinese community pointed out in the report of the integration of e-commerce to achieve a basic function for Chinese community provides excellent opportunities, electronic commerce will provide broad prospects for the development of Chinese community.

\section{Community e-commerce system based on J2EE}

Java Servlet API provides a standard way to extend any function that uses a server based on request / response protocols. Servlet is the server-side Java code, its essence is a Java class, mainly used in Web server. On any Web server that supports Servlet, you can process requests and generate dynamic Web content. Servlet has the following features:

(1) combining with HTTP protocol, almost all requests of HTTP protocol can be controlled, and it is easy to be developed by encapsulation.

(2) support multithreading, and always stay in memory after the first call, fast response and less consumption of resources.

(3) have all the advantages of Java applications (such as portability, robustness, easy development, etc.), and access to all API on the Java platform.

(4) the program design can be done by using the object oriented design idea.

(5) its program is modular, each program performs a specific task, and can exchange information through pipeline flow, service global variables, page redirection and other technologies, and then work together.

In addition, Servlet uses the method of putting user information on the server to solve the problem of session management, and overcomes the problems of poor confidentiality, small data capacity, and influenced by customer settings.

However, Servlet uses HTML statements to dynamically implement web pages. The combination of dynamic and static parts of the page is very complicated and confusing, which leads to writing difficulties (which is the reason for the emergence of JSP).

In order to make up for the disadvantages of Servlet, Sun has developed JSP technology [5-6] on the basis of it. JSP technology provides a simple and fast method for creating dynamic content of Web pages. The purpose of the design is to make it easier and more convenient to build Internet 
based applications.

JSP is a senior, friendly and similar to the HTML tag type interface, is embedded in the HTML code of Java code, the code can accomplish many functions (such as access database, send E-mail, display data etc.). When the user requests the JSP page, the Web server first compiles it into a class similar to Servlet, then instantiate the class and respond to the user request, and finally return the result to the client layer.

JDBC API accesses a variety of databases in a unified manner, and its interfaces are contained in the two packages of java.sql and javax.sql. JDBC isolates the problems between developers and private databases, and JDBC can also provide platform independent database access because of the Java implementation of the mechanism.

JNDI is used to execute naming and directory services, and its interfaces are contained in javax.naming and its sub packages. JNDI provides standard directory manipulation methods for applications (such as getting the associated attributes of objects, searching objects according to their attributes, etc.). Through JNDI, J2EE applications can store and dynamically retrieve any type of named Java object.

JNDI is divided into two parts: application programming interface (API) and the service provider interface (SPI), which allows Java applications to access various naming and directory services, the latter is a kind of service for any suppliers (including the use of directory service provider).

JavaBean is a new component technology designed specifically for current software developers. It provides an excellent solution for software developers. The prominent feature of JavaBean is in one word: "Write once, Run anywhere, Reuse everywhere", that is, "once written, executed anywhere, reused anywhere."'. This feature is actually a solution to the growing complexity of the troubled software industry, providing a simple, compact, and excellent solution for software developers.

We can see the JavaBean as a black box (i.e., only need to know its function without regard to the internal structure of the software, the black box only) is introduced and defined its external features and the interface with other parts. JavaBean provides 3 interfaces to the outside:

(1) the method that JavaBean can call.

(2) readable and writable attributes provided by JavaBean.

(3) JavaBean sends or receives events from outside.

\section{Community E-commerce}

Online shopping mall system is an important part of the community e-commerce platform, it mainly to their own business platform without selling platform (the franchisee) as well as the community itself to provide a variety of service management. Its main business functions include: commodity management, information release, shopping guide promotion, business management, marketing statistics, value-added services, search engines, membership management, operation support, basic organizational structure, etc..

In the future development of the system, there must be an increasing amount of browsing. In order to meet the demand of large users in the future, we must consider the problem of how to expand the future system in the selection of our system. In this system, we use the system cluster technology to solve the expansion of the system capacity. The so-called system cluster, simply said: is a number of servers at the same time for a system to provide the same service. The system from two aspects of Web server cluster technology and database cluster technology to consider.

(1) Web server cluster technology

Now the mainstream Web server mainly includes BEA WEBLOGIC, IBM WEBSPHERE, JBOSS, RESIN, TOMCAT and so on. Among them, JBOSS, RESIN, TOMCAT is widely used, and is a free Web application server. The website system is based on different Web servers, and adopts the following cluster technologies:

(1)Main / main (Active/active)

This is the most commonly used cluster model, and it has high availability. The model allows 
maximum utilization of hardware resources. Each node through the network to the client to provide resources, each node capacity is predefined, so to achieve optimal performance, and each node can be temporarily taken over by another node in failovers work. All services remain available after failover, but performance generally declines.

(2)Master / slave (Active/passive)

In order to provide maximum usability, and the influence on the performance of the minimum, the Active/passive model requires a standby in the normal work of the spare contact, the master node processing client requests and standby node in the idle state, when the master node fails, standby node will take over the main node, continue to provide services for the client, and will not have any impact on performance.

(3)Mixed type (Hybrid)

Hybrid is the combination of the above two models, only for critical applications for failover. It ensures that the application can be implemented on the server as well as the availability of non critical applications. When failures occur, less critical applications on the fail server can be used, but those critical applications can be transferred to another available node to achieve balance between performance and fault tolerance in two ways.

(2) database cluster technology

Database cluster [17] simply understand: a series of database collections, and these databases can be accessed through a single database server instance.

\section{Summary}

In this system, we mainly use the database itself cluster technology to combine the software architecture of the system for configuration and application. For example, the database uses Oracle, and the database cluster can use Oracle RAC. RAC based e-commerce application users or middle tier application server clients can be connected to the database through virtual database service name. Oracle automatically balances user loads between multiple nodes in a cluster. Real Application Clusters database instances on different nodes reserve all database services or partial subset database services. This makes DBA highly flexibly connected to the specific application of customer specific database services, and whether it is easier to identify the application client can connect to the database node of some or all of the. RAC during work, each node can be used alone (and balanced by application load). If an accident occurs, such as a failure of a node, the failover of the node can be implemented to ensure the high availability of the database $24 * 7$.

\section{References}

[1] Pahlavan K, Krishnamurthy P and Geng Y, Localization challenges for the emergence of the smart workd. IEEE Access, 2015, 3(1), pp. 3058-3067

[2]Lv, Z., Tek, A., Da Silva, F., Empereur-Mot, C., Chavent, M., \&Baaden, M. (2013). Game on, science-how video game technology may help biologists tackle visualization challenges. PloS one, 8(3), e57990.

[3] Lv, Z., Halawani, A., Feng, S., Li, H., \&Réhman, S. U. (2014). Multimodal hand and foot gesture interaction for handheld devices. ACM Transactions on Multimedia 